Supporting Information

\title{
Quantification of Multivalent Interactions by Tracking Single Biological Nanoparticle Mobility on a Lipid Membrane
}

Stephan Block ${ }^{1}$, Vladimir P. Zhdanov ${ }^{1,2}$, Fredrik Höök ${ }^{1}$

1 Division of Biological Physics, Department of Physics, Chalmers University of Technology, SE-412 96 Gothenburg, Sweden

${ }^{2}$ Boreskov Institute of Catalysis, Russian Academy of Sciences, Novosibirsk 630090, Russia

Contents

S1. Concepts behind the trajectory filtering approach

S2

S2. Fluctuations of the time-resolved diffusion coefficient $\quad$ S6

S3. Illustration of trajectory filtering S6

$\mathrm{S} 4$. Linear versus logarithmic binning in the $D$ histograms $\quad \mathrm{S} 7$

S5. Filtered $D$ histograms for real SPT data $\quad$ S9

S6. Free draining model vs. the Saffman-Delbrück model S9

S7. Extraction of transition rates $\quad \mathrm{S} 15$

S8. Monte Carlo validation of the free draining model $\quad$ S23

S9. Width of $n$ distribution $\quad$ S25

S10. Materials and methods $\quad$ S26 


\section{S1. Concepts behind the trajectory filtering approach}

In this section, we show that particles, which change their diffusion coefficient, $D$, over the course of their trajectory, exhibit an increase in the intrinsic fluctuations of their time-resolved diffusion coefficient, $D(t)$. This destroys any peak structure in the $D$ histograms (as expected if subpopulations exist that possess distinct $D$ values). However, analysing the fluctuations of $D(t)$ allows to identify and remove such nonsingle $D$-value trajectories from further data analysis, restoring the peak structure in a $D$ histogram. Following this line, we first recall the statistical properties of random walks, which is necessary to understand the filtering concept, and then test the filtering concept using synthetic SPT movies (derived from stochastic simulations).

During a two-dimensional random walk, the probability to observe a particle displacement $d$ after a time lag $\Delta t$ is given by

$$
p(d, \Delta t)=\frac{1}{\sqrt{\pi \cdot 4 D \Delta t}} \cdot \exp \left(-\frac{d^{2}}{4 D \Delta t}\right)
$$

where $4 D \Delta t$ is the mean squared displacement (MSD). ${ }^{1}$ In single particle tracking (SPT), MSD of a certain track for a given lag time $\Delta t$ is usually determined using the approach of internal averaging, ${ }^{2}$ which is reduced to calculation of the squared displacement of all particle positions of the track being separated by the time lag $\Delta t$, followed by averaging these squared displacements. This procedure is repeated for different lag times $\Delta t$, which are typically chosen to be multiples of the frame rate $1 / \Delta t_{0}$ of the SPT movie, $\Delta t=m^{*} \Delta t_{0}$. For a random walk, this yields MSD $=4 D \Delta t_{0} m$. The slope of this linear dependence of MSD on $m$ allows extraction of $D$ using a linear fit (Figure 1e).

It was recently shown that the accuracy of this extraction procedure is governed by the length of the trajectories and the accuracy in localizing the particle positions. ${ }^{3}$ Neglecting localization error, it is established that the extracted diffusion coefficient $D$ varies (due to the finite trajectory length) around the true value with the normalized standard deviation

$$
\frac{\sigma}{D}=\sqrt{\frac{2}{3} \cdot \frac{N_{\mathrm{p}}}{N-N_{\mathrm{p}}}} \quad \text { (for } N>>N_{\mathrm{p}} \text { ) }
$$



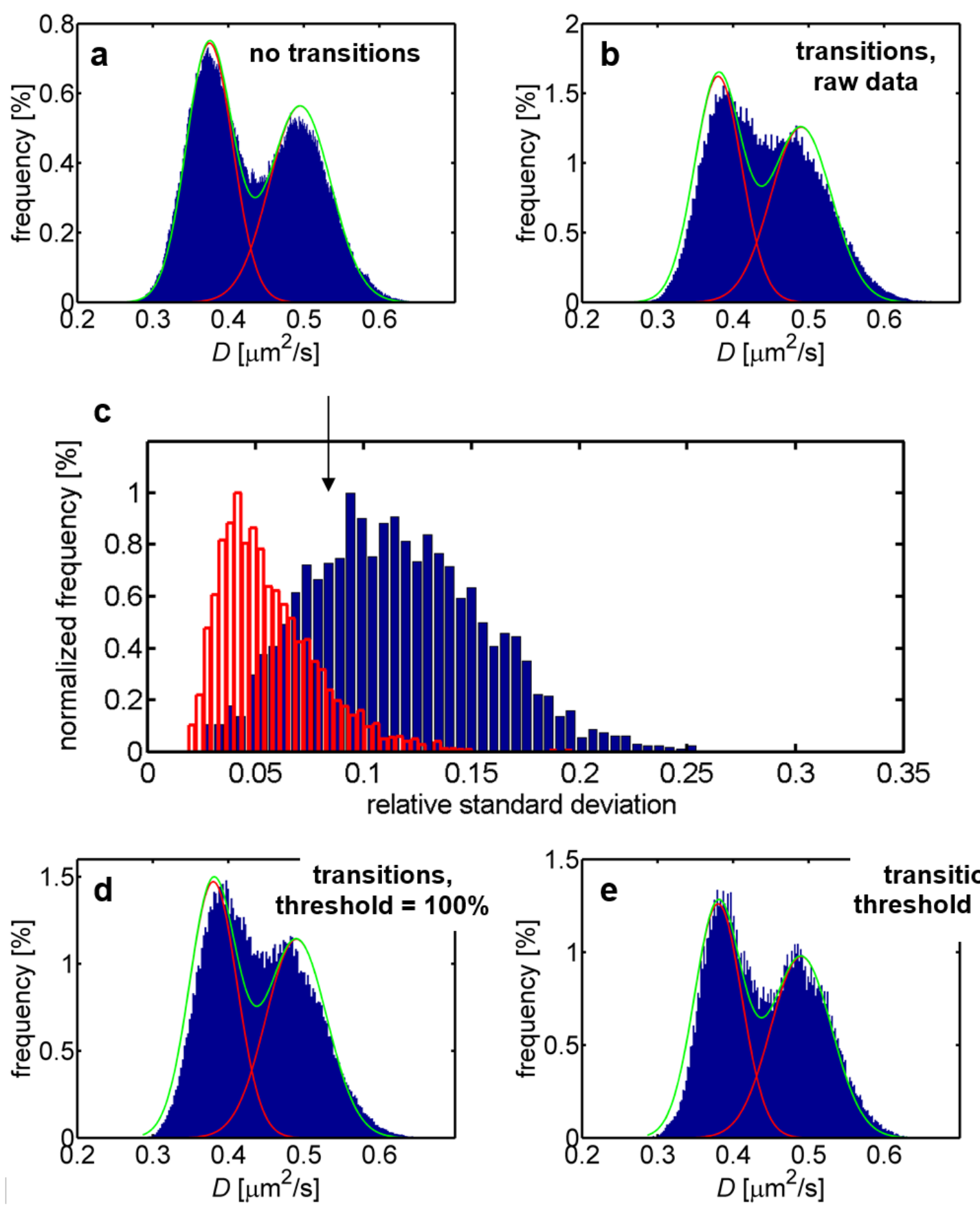

Figure S1: Histograms of the average values $(\mathbf{a}, \mathbf{b}, \mathbf{d}, \mathbf{e})$ and relative standard deviations of the timeresolved diffusion coefficients extracted from synthetic SPT movies $\left(N_{0}=500\right.$ frames, $N=200$ moving window). Red solid lines give the theoretically expected distributions of the extracted diffusion coefficients according to Equation S2, while the green line shows their superposition. In histogram (a), the two peaks extracted from the movies correspond to two particle populations having a diffusion coefficient of either 0.375 or $0.5 \mu \mathrm{m}^{2} / \mathrm{s}$ throughout the entire movie. However, addition of a third particle population (having a diffusion coefficient making a transition from 0.375 to $0.5 \mu \mathrm{m}^{2} / \mathrm{s}$ in the middle of the SPT movie) distorts the peak structure in the histogram. Analysis of the relative standard deviation of the time-resolved diffusion coefficients reveals (c) that most trajectories exhibiting a transition between 2 diffusion coefficients (blue bars) have larger relative standard deviations than expected from Equation S2 (black arrow), while most single diffusion coefficient trajectories showed a distribution (red bars) whose average value is close to the expectation value given by Equation S2. Rejecting all trajectories whose relative standard deviations exceeds a certain threshold $[(\mathbf{d})=100 \%$ of Equation S2, (e) $=75 \%$ of Equation S2] preferentially removes particles from the data analysis that have a non-constant diffusion coefficient and restores the initial peak spectrum in the histogram. 
if the procedure is performed on a trajectory having $N$ frames and if $D$ is determined by a linear fit of MSD for $n$ ranging between 0 and $N_{\mathrm{p}}$ (i.e., involving a maximum time lag $\left.N_{\mathrm{p}}{ }^{*} \Delta t_{0}\right){ }^{2},{ }^{4}$ This relative standard deviation is independent of $D$. In fact, Equation S2 is the dominating source of uncertainty if the particle's MSD is larger than the localization accuracy (which can usually be experimentally achieved by lowering the acquisition rate).

Equation S2 can be used to identify those particles, whose diffusive coefficient changed in the course of the trajectory. Consider a SPT experiment having 2 particle populations that have 2 distinct $D$ values. According to Equation $\mathrm{S} 2$ it is theoretically possible to determine $D$ with sufficient accuracy to observe 2 distinct peaks in a $D$ histogram, if the length $N$ of the analysed trajectories exceeds a cut-off value (which can be estimated from Equation S2). For example, for 2 populations, one of which having a diffusion coefficients of 0.5 and the other of $0.375 \mu \mathrm{m}^{2} / \mathrm{s}$, it is (using $N_{\mathrm{p}}=2$ ) sufficient to include trajectories having $N>200$ frames to resolve these 2 peaks (Figure S1a). This follows directly from Equation S2, which under these conditions predicts a normalized standard deviation of $8 \%$,which is much smaller than the normalized peak distance of $28 \%(=(5-3.75) /(5 / 2+3.75 / 2))$.

In real experiments, however, trajectories may get distorted, e.g. due to transient sticking of a particle to contaminations / imperfections or due to changes of the number of linkers, causing transient changes in the particle mobility. Especially the latter is very likely to occur for multivalent interactions, e.g. for virions or vesicles that transiently change the numbers of bound receptors. Both mechanisms transiently change $D$ in the course of the trajectory and application of internal averaging over the whole trajectory extracts ill-defined $D$ values that usually deviate from the real values.

This is illustrated in Figure S1b showing 3 particle populations: the 2 populations from Figure S1a having diffusion coefficients of 0.5 and $0.375 \mu \mathrm{m}^{2} / \mathrm{s}$, plus a third population of particles undergoing a transition in $D$ from 0.375 to $0.5 \mu \mathrm{m}^{2} / \mathrm{s}$ in the middle of the SPT trajectory. Application of internal averaging on these particles gives an (ensemble averaged) $D$ value of $0.4375 \mu \mathrm{m}^{2} / \mathrm{s}$, which adds a third peak to the $D$ histogram and thereby wipes out the initial peak structure (Figure S1b). As SPT experiments will always contain a certain fraction of trajectories that have a nonconstant diffusion coefficient, it is clear that such trajectories have to be isolated from the data pool, as their inclusion in the $D$ histogram destroys any peak structure that would be indicative for populations having distinct $D$ values.

Our approach to exclude such non-single diffusion coefficient trajectories relies on a subtrajectory analysis that uses a rolling window to restrict the internal averaging procedure to a (continuous) subset of the full trajectory, yielding a timeresolved $D(t)$ for the time span covered by the rolling window. Shifting the rolling window over the whole trajectory therefore allows to determine the temporal behaviour of $D$. For example, for a trajectory covering $N_{0}$ consecutive frames and a rolling-window size of $N$ frames, this approach will yield $N_{0}-N+1$ subtrajectories by 
considering only the frames 1 to $N, 2$ to $N+1$, etc, leading to $N_{0}-N+1 D$ values that are temporally resolved over the course of the trajectory.

For an undisturbed random walk having a constant $D$ over the whole trajectory, a rolling window of $N$ frames will extract a time-resolved diffusion coefficient that fluctuates with a relative standard deviation given by Equation S2 around the true value (if other noise sources like localization noise can be neglected). However, if the initial trajectory is composed of two or more diffusion coefficients, the fluctuations of the extracted diffusion coefficient will be caused by two processes, the stochastic fluctuations of a random walk and additionally transitions between different diffusion coefficients. Hence, the relative standard deviation of the diffusion coefficient will exceed the expectation value given by Equation S2, which includes only the stochastic fluctuations. It is therefore straightforward to calculate the expected relative standard deviation based on the parameters of the subtrajectory analysis $\left(N_{\mathrm{p}}\right.$ and $\left.N\right)$ and to filter out all trajectories whose time-resolved $D(t)$ exhibits a relative standard deviation exceeding the expectation value given by Equation S2.

This concept is illustrated in Figure S1c-e, showing histograms of the relative standard deviation of single and non-single diffusion coefficient trajectories (Figure $\mathrm{S} 1 \mathrm{c}$ ) and the evolution of the $D$ histogram (Figure S1d, e), if more and more distorted trajectories are excluded by decreasing the rejection threshold (measured relative to the expectation value Equation S2). These plots are based on the same synthetic SPT movies used in Figure S1b, which contain a mixture of single diffusion coefficient trajectories (having either 0.375 or $0.5 \mu \mathrm{m}^{2} / \mathrm{s}$ ) and trajectories undergoing a transition from 0.375 to $0.5 \mu \mathrm{m}^{2} / \mathrm{s}$ in the middle of the trajectory. These trajectories were again analysed using a rolling window having $N=200$ frames and internal averaging with $N_{\mathrm{p}}=2$ (Figure S1c), leading to an expected relative standard deviation of $8 \%$ for single diffusion coefficient trajectories.

The relative standard deviation extracted for single diffusion coefficient trajectories (Figure S1c, red bars) showed a distribution having an average value being close to the expected value of $8 \%$ as given by Equation 3 (black arrow in Figure S1c). In contrast, this distribution shifted to much larger values for the nonsingle diffusion coefficient trajectories (Figure S1c, blue bars), confirming that most of these trajectories have relative standard deviations exceeding the expectation value for single diffusion coefficient trajectories. Therefore it was investigated how exclusion of trajectories, whose relative standard deviation exceeds a certain rejection threshold, from further data analysis affects the measurements statistics and the distribution of the extracted diffusion coefficients. Choosing the expectation value of Equation S2 as threshold, $13 \%$ of the single but $77 \%$ of the non-single diffusion coefficient trajectories were removed. These values increased to $34 \%$ and $91 \%$ for lowering the rejection threshold to $75 \%$ of Equation S2, and finally $45 \%$ and $94 \%$ at $66 \%$ of Equation S2.

Although exemplary in nature, the calculation shows that the proposed filtering procedure preferentially removes transition containing trajectories rather than single- 
diffusion coefficient trajectories from the data analysis. This behaviour reproduced well in simulations using different parameters. The preferential rejection of non-single diffusion coefficient trajectories is advantageous, as the peak spectrum becomes resolvable again in the $D$ histogram (see Figure $\mathrm{S} 1 \mathrm{~b}, \mathrm{~d}$, e for the evolution of $D$ histograms for different filtering / rejection strength).

\section{S2. Fluctuations of the time-resolved diffusion coefficient}

In Section S1, we have shown that the relative standard deviation of time-resolved $D(t)$ increases if the underlying trajectory is subject to transient changes in the diffusion constant. Hence, a high relative standard deviation (with respect to the expectation value of Equation S2) indicates a high probability that such transitions occurred during the course of the trajectory. This feature should therefore be used in further data analysis.

This can be implemented in the data analysis by using histograms of the pooled time-resolved diffusion coefficients instead of histograms that were created from trajectory-averaged diffusion coefficients. In the latter case, one loses the information of the (relative) standard deviations as only average values enter into the histogram. However, if one uses the time-resolved diffusion coefficients instead, events are added to the histogram that are narrowly distributed around the position of the respective average values (small standard deviations) for trajectories having no transient changes in the diffusion coefficient, while occurrence of transient changes increases the standard deviation and therefore distributes the events over a broader interval. Hence, well-defined diffusion coefficients will add narrow peaks to the histogram, while less well-defined diffusion coefficients (e.g., due to the presence of transitions) will add broad peaks, giving less structure to the histogram.

In the ideal case, in which all trajectories are free of artefacts, this approach simply replaces each diffusion coefficient (averaged over the whole trajectory) by a Gaussian distribution that is centred at the same average value, but which has a standard deviation that is indicative for the measurement resolution.

\section{S3. Illustration of trajectory filtering}

In a next step it was tested, how the trajectory filtering procedure modifies the $D$ histogram of a SPT experiment that has (as input parameter) a quasi-continuous distribution of diffusion coefficients. To be applicable in real experiments, one has to be sure that the occurrence of peaks in the $D$ histogram results from presence of particle populations differing in their diffusion coefficient and not from an erroneous modification of an initial quasi-continuous diffusion coefficient distribution by the filtering process. Or in other words, quasi-continuous distributions should remain quasi-continuous after application of the filtering process, while occurrence of a peak spectrum should always require that the initial diffusion coefficient distribution consisted of distinct peaks. 
This was investigated by creating synthetic SPT trajectories. In the first case (quasi-continuous distribution), diffusion coefficients were selected from an interval ranging between 0 and $0.5 \mu \mathrm{m}^{2} / \mathrm{s}$ using a step size of $0.002 \mu \mathrm{m}^{2} / \mathrm{s}$ and one trajectory was generated for each of these diffusion coefficients. As the spacing between the diffusion coefficients is much smaller than the resolution of a typical SPT analysis, this SPT experiment possesses a quasi-continuous distribution of diffusion coefficients. In the second case (discrete distribution), 75 trajectories were generated having diffusion coefficients of either $0.5,0.33,0.25$ or $0.2 \mu \mathrm{m}^{2} / \mathrm{s}$. Contrary to the first case, the peak spacing is sufficiently large so that individual peaks are resolvable using a rolling window of $N=200$ and internal averaging up to $N_{\mathrm{p}}=2$.

These trajectories were analysed as described in Section $\mathrm{S} 1$ and $D$ histograms were created for different filtering strengths (Figure S2). It is apparent from these histograms that even at high filtering strengths (50\% of Equation S2) the filtering procedure does not "cut peaks" into the quasi-continuous distribution of diffusion coefficients (Figure S2, left), while a peak structure is only observed after filtering, if the input distribution of the diffusion coefficients consisted of distinct peaks (Figure S2, right).

\section{S4. Linear versus logarithmic binning in the $D$ histograms}

Equation S2 shows that the relative scattering of $D$, which is extracted using the internal averaging procedure, depends only on the length $N$ of the trajectory and maximum time lag $N_{\mathrm{p}}{ }^{*} \Delta t_{0}$ used during the internal averaging. This means that (for identical measurement conditions) a population of particles having a small diffusion coefficient creates a much more narrow distribution in the diffusion coefficient histogram than a population with a much larger diffusion coefficient, simply because the relative standard deviation of these distributions is fixed (Figure S2d, right). For a system that has several populations with diffusion coefficients being distributed over a large interval, it can be problematic to identify all peaks, since the slow diffusion coefficients create much more narrow peaks than the fast ones.

This problem is solved by creating the histograms using a logarithmic binning of the diffusion coefficient. In such a plot, all peaks have the same width, making their identification easier (Figure S2e, right). Moreover, as most theories predict either a logarithmic (e.g., Saffman-Delbrück-model) ${ }^{5}$ or inverse (e.g., free draining model) $)^{6,7}$ relationship between diffusion coefficient and the number of links, the use of a logarithmic binning allows to easily compare the peak positions between experiments, in which the number of links is varied. 

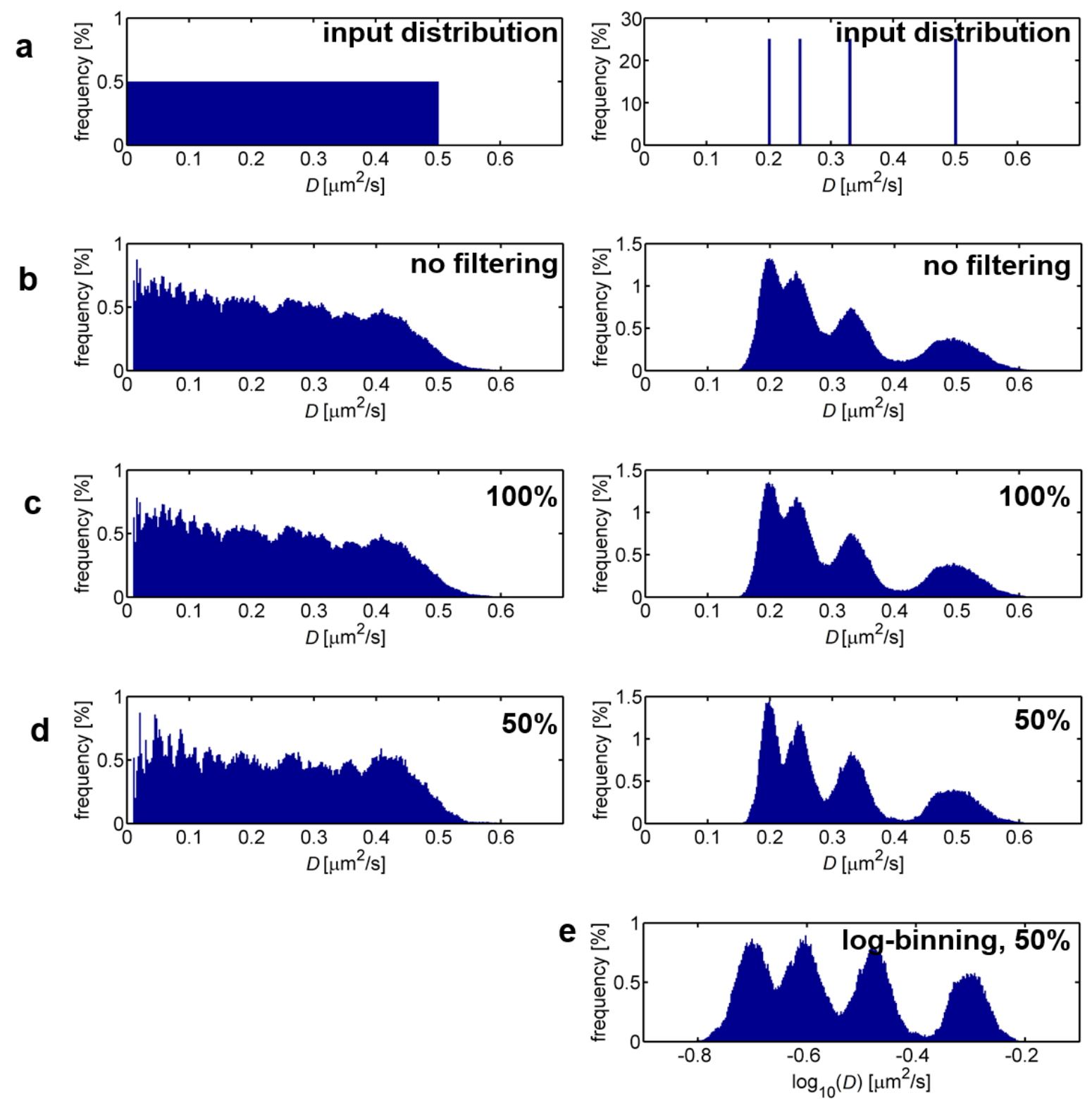

Figure S2: Trajectory filtering applied to a quasi-continuous (left) and discrete spectrum (right) of diffusion coefficients. The top row (a) shows the diffusion coefficient distributions used to create synthetic TIRFM SPT movies, while rows $\mathbf{b}-\mathbf{e}$ give the extracted distributions without any trajectory filtering (b), or for different rejection thresholds as indicated (c-e). Note that the peaks have the same width in a logarithmic binning (e), as their standard deviation normalized by the peak position is constant (Equation S2). 


\section{S5. Filtered $D$ histograms for real SPT data}

Figure 2 in the main part of the article showed that application of the trajectory filtering procedure to SUV trajectories indeed creates $D$ histograms that clearly exhibit distinct peaks, which are indicative for populations of SUVs differing in their $D$ value. It is instructive to investigate, how the $D$ histogram evolves for increasing filtering strength (see Figure S3 using the data shown in Figure 2b).

Without any filtering, the peak spectrum is only barely visible (Figure S3a). Filtering out all trajectories with relative standard deviations exceeding the expectation value given by Equation S2 (i.e., 100\% of Equation S2; Figure S3b) preferentially rejects trajectories having apparent diffusion coefficients between 0.01 and $0.1 \mu \mathrm{m}^{2} / \mathrm{s}$, which are most likely caused by SUVs that temporarily stick to imperfections, leading to an underestimation of the actual trajectory diffusion coefficients. A further increase in the filtering strength $(75 \%$ and $60 \%$ of Equation S2; Figure S3c and S3d, respectively) starts to reject trajectories having diffusion coefficients between 0.1 and $1 \mu \mathrm{m}^{2} / \mathrm{s}$, revealing the actual peak structure in the $D$ histogram. These filtered trajectories are likely to contain transitions between the distinct tethering states (which form the distinct peaks in the $D$ histogram) as their inclusion "fills up the valleys" between the peaks in the $D$ histogram (see section $\mathrm{S} 1$ ). Note that each of these peaks is formed by several trajectories, indicating that trajectories constituting a certain peak must have very similar $D$ values; otherwise the peak would be too broad to be resolvable in the $D$ histogram. As the peak positions reproduce well between independent measurements (see e.g. dashed lines in Figures 2 and 3) and since the filtering procedure itself is not able to cut such peaks in a quasi-continuous $D$ histogram (Section S3), one can rule out that the observed peak spectrum is a statistical artefact caused by the filtering process.

\section{S6. Free draining model vs. the Saffman-Delbrück model}

The good reproducibility of the peak positions throughout the different $D$ histograms motivated us to ask whether the data is possible to describe using the model of Saffman and Delbrück, ${ }^{5}$ or if rather the so-called free draining model describes the data best. ${ }^{6,7}$

The Saffman-Delbrück model quantifies the diffusion coefficient $D$ of a cylindrical inclusion (radius $r_{\text {in }}$ ), which completely protrudes through a membrane having a $2 \mathrm{D}$ viscosity $\eta_{\mathrm{m}}$. The membrane is assumed to be immersed in a fluid (bulk viscosity $\eta_{\mathrm{fl}}$ ) from both sides. In that case, the diffusion coefficient $D$ is given by

$$
D=\frac{k_{\mathrm{B}} \cdot T}{4 \pi \cdot \eta_{\mathrm{m}}} \cdot\left[\ln \left(\frac{\eta_{\mathrm{m}}}{\eta_{\mathrm{fl}} \cdot r_{\mathrm{in}}}\right)-\gamma\right],
$$




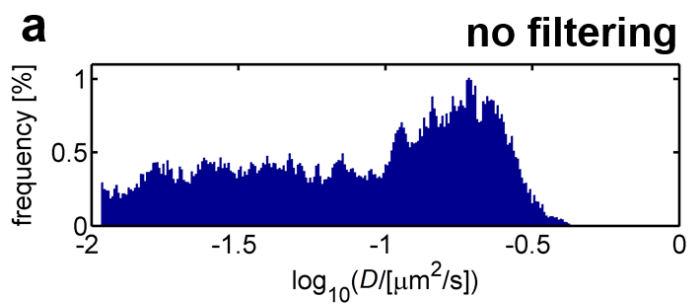

b
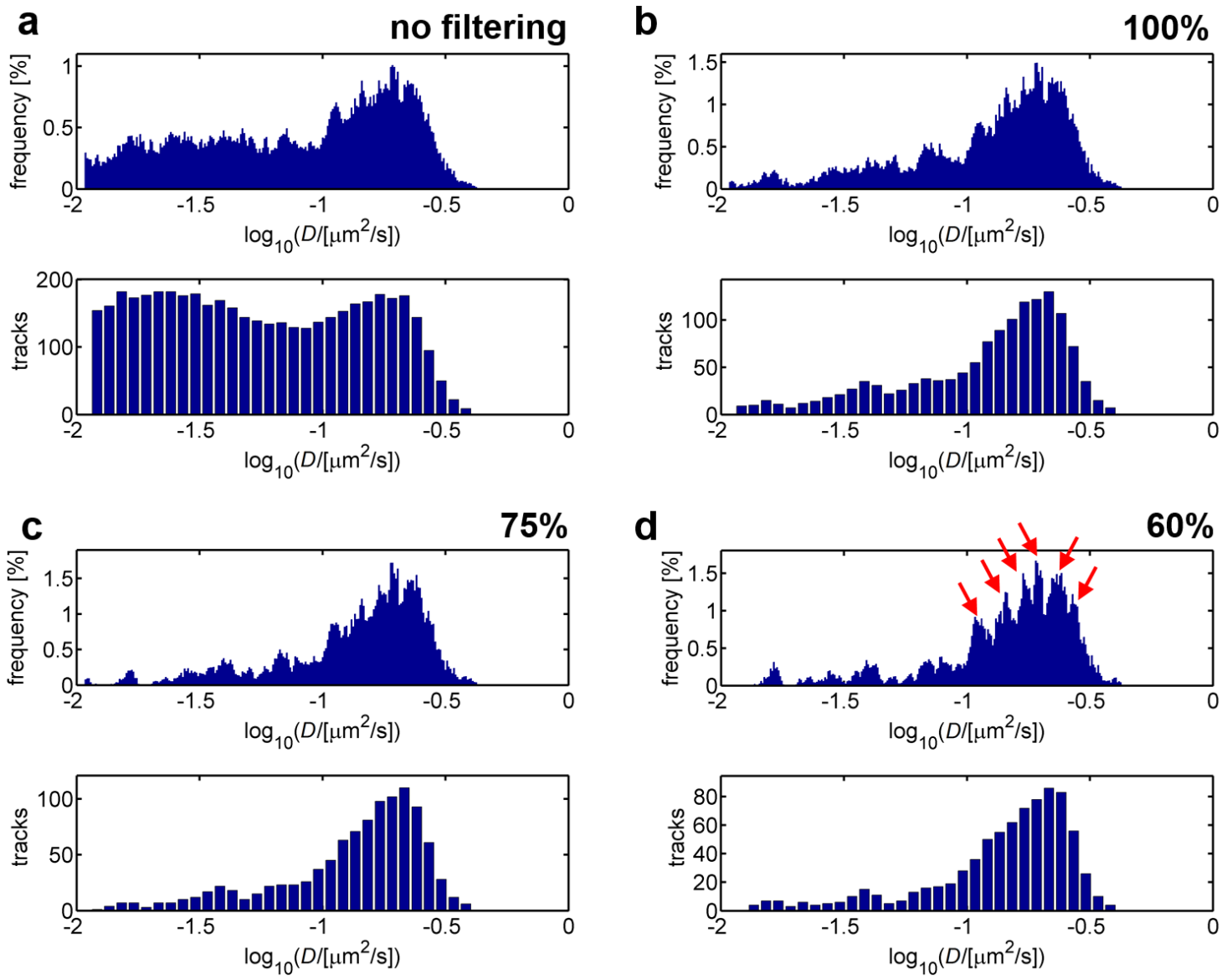

Figure S3: Evolution of the diffusion coefficient histogram of Figure $2 \mathrm{~b}$ with increasing filtering strength. The indicated rejection thresholds are expressed with respect to the expectation value, Equation S2 (e.g. in d, all trajectories were removed from data analysis whose relative standard deviation exceeded $60 \%$ of the expectation value given by Equation S2). The upper panels show the diffusion coefficient histogram using a logarithmic binning, while the lower panels give the number of trajectories that contribute to a particular region of the histogram indicated by the bin. The red arrows in $\mathbf{d}$ indicate the positions of the red dashed lines in Figure 2.

where $\gamma=0.577$ is the Euler- Mascheroni constant. The inclusion radius of a cylindrical aggregate that is formed by $n$ DNA-tethers (linking the vesicle to the SLB) can be approximated by

$r_{\text {in }}=r_{0} \cdot \sqrt{n}$.

where $r_{0}$ denotes the inclusion radius of a single DNA-tether. Recent publications reported values for the $2 \mathrm{D}$ membrane viscosity $\eta_{\mathrm{m}}$ ranging between $10^{-9}$ and $10^{-8} \mathrm{~Pa}$ $\mathrm{m} \mathrm{s}^{8-11}$ Moreover, using a water viscosity of $\eta_{\mathrm{fl}}=10^{-3} \mathrm{~Pa} \mathrm{~s},{ }^{12}$ and an single DNAtether inclusion radius of $r_{0}=1 \mathrm{~nm},{ }^{13}$ it follows that the logarithmic term in Equation 
S3 exceeds the Euler- Mascheroni constant by one order of magnitude, allowing to approximate Equation S4 by

$$
D=\frac{k_{\mathrm{B}} \cdot T}{4 \pi \cdot \eta_{\mathrm{m}}} \cdot\left[\ln \left(\frac{\eta_{\mathrm{m}}}{\eta_{\mathrm{fl}} \cdot r_{0}}\right)-\frac{1}{2} \ln (n)\right] .
$$

This creates a very weak dependence of $n$ on $D$, yielding positions of the diffusion coefficient peaks, which are far too close to be resolvable with the accuracy achieved in this study. In other words, if the assumptions of the Saffman-Delbrück model would apply to DNA-tethered vesicles, the $D$ histogram cannot show distinct peaks as neighbouring peaks would be too close to each other to be independently resolvable. It is therefore concluded that the data are incompatible with the model of Saffman and Delbruck.

As shown in the main part of the article, the peak spectra are well described by the so-called free draining model, which connects the vesicle diffusion coefficient $D$ with the number $n$ of linking DNA-tethers, and the diffusion coefficient of a single DNA-tether $D_{*}: 6,7$

$$
D=\frac{D_{*}}{n} \Rightarrow \frac{1}{D}=\frac{n}{D_{*}}=\frac{n_{\mathrm{p}}+n_{\mathrm{p}, 0}}{D_{*}} .
$$

The analysis, which was done for one representative case in the main manuscript, is given for all experiments in Figure S4. The peak positions entering the analysis are marked by red dashed lines in the diffusion coefficient histograms. For all experiments, a linear relationship between the inverse peak diffusion coefficient $1 / D$ and the peak (observation) number $n_{\mathrm{p}}$ (according to Equation S6) is observed (Figure $\mathrm{S} 4$, right column). Fitting Equation $\mathrm{S} 6$ allowed to extract the diffusion coefficient of a single DNA-tether $D_{*}$ and the displacement $n_{\mathrm{p}, 0}$ (relating $n_{\mathrm{p}}$ with $n$ ); both values are given in Figure S4. Finally, averaging the diffusion coefficients of peaks having the same value of $n$ enabled to retrieve the peak diffusion coefficients with higher accuracy. These refined peak diffusion coefficients are given by dashed blue lines in the histograms of Figure S4, allowing an easy comparison with the observed peak positions (indicated by the red dashed lines). 

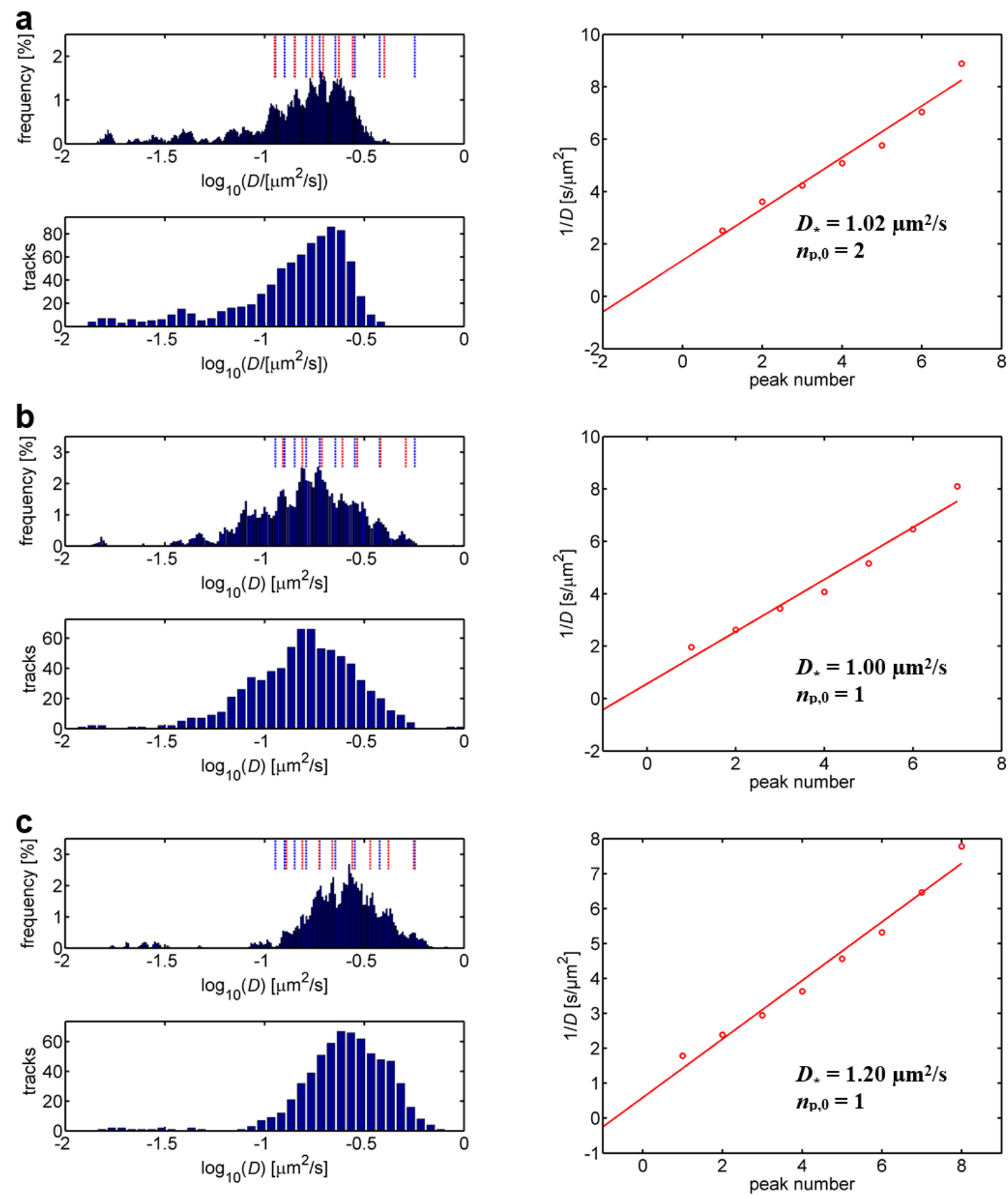

Figure S4a-c: Diffusion coefficient histograms of SUVs linked on average via 3 DNA-tethers (left, top panel; as estimated from the DNA and vesicle concentrations used during the self-assembly process and the assumption of $100 \%$ insertion efficiency), number of trajectories that contribute to a particular region of the histogram indicated by the bin (left, bottom panel) and plot of the inverse peak diffusion coefficient versus the respective peak observation number (right) as motivated by the free draining model (Equation S6). Peaks included in the data analysis are marked by a red dashed line in the diffusion coefficient histograms, while the blue dashed lines indicate the position of the refined diffusion coefficients (obtained by averaging the diffusion coefficients of a particular peak, observed in the different measurements; see Table S1), allowing a comparison of peak positions between the different histograms of this figure. The red line is a linear fit of the inverse peak diffusion coefficient according to Equation S6, yielding the diffusion coefficient of a single DNA-tether $D_{*}$ and the displacement $n_{\mathrm{p}, 0}$ (relating $n_{\mathrm{p}}$ with $n$ ) as indicated in the right column. 

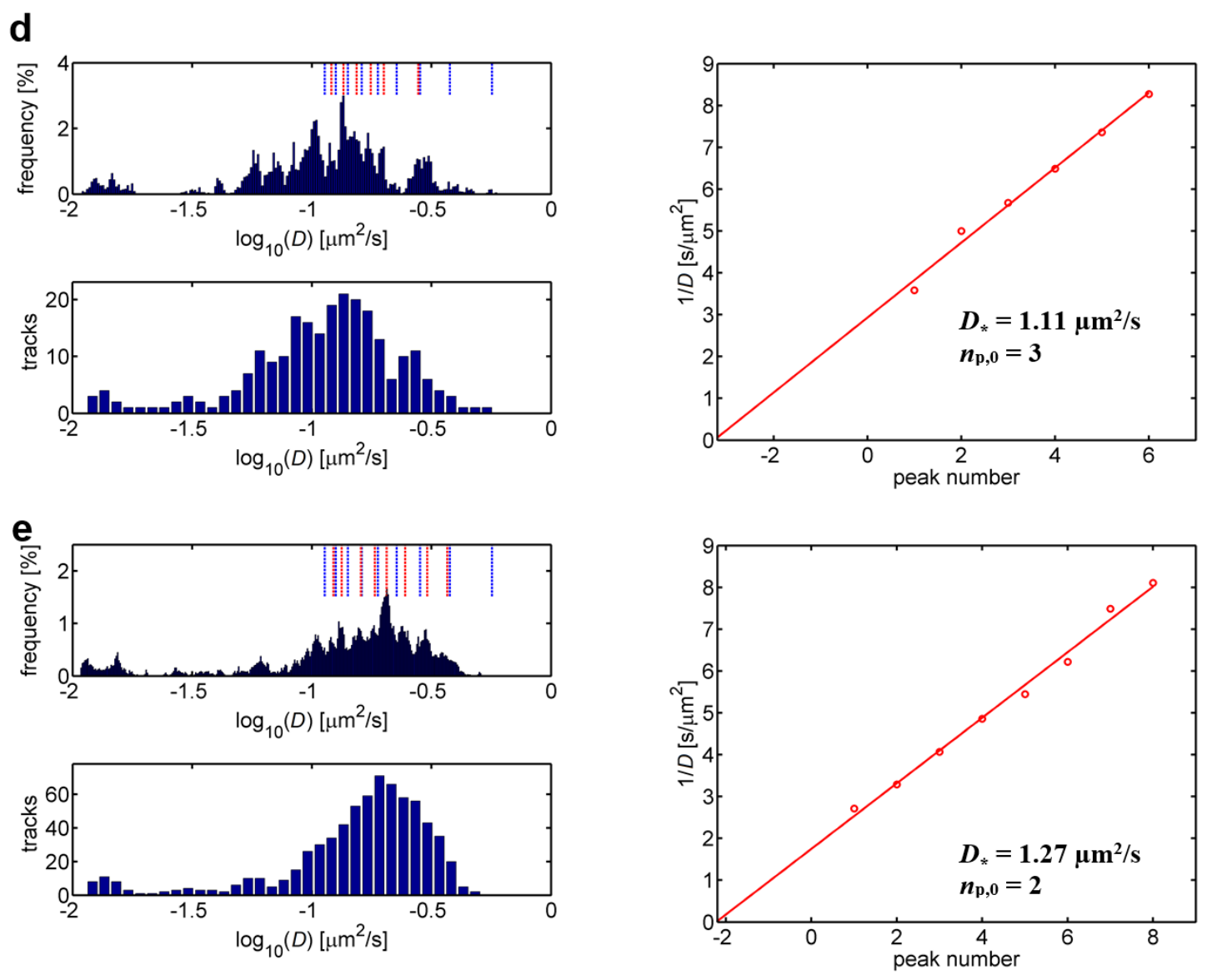

Figure S4d-e: As Figure S4a-c for SUVs linked on average via 6 DNA-tethers. 

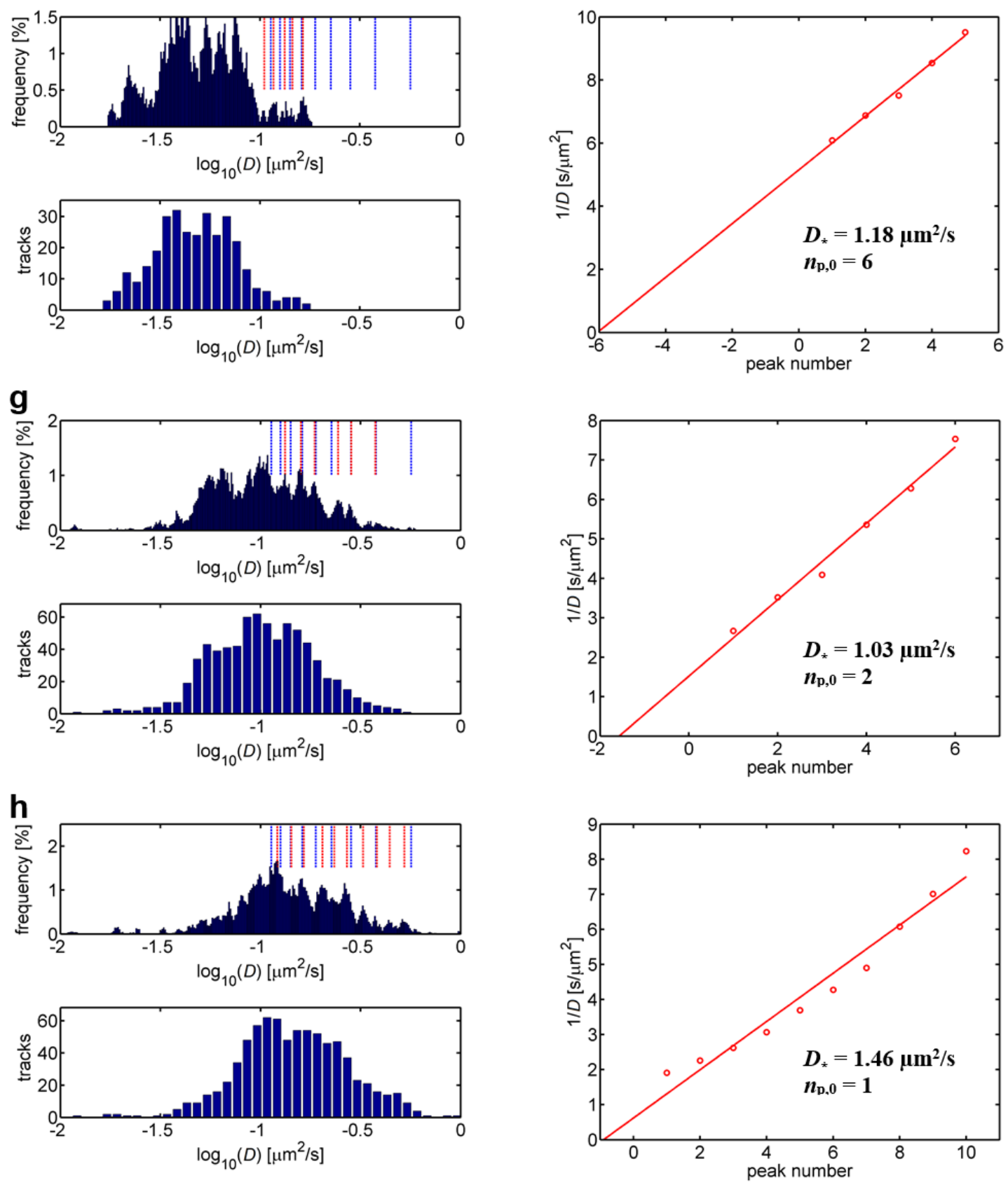

Figure S4f-h: As Figure S4a-c for SUVs linked on average via 9 DNA-tethers. 


\section{S7. Extraction of transition rates}

Succeeding in identification of the relation between vesicle diffusion coefficient $D$ and the number $n$ of DNA-tethers linking this vesicles to the SLB (Figure 4), we were curious to also explore the possibility of relating fluctuations in the time-resolved diffusion coefficient $D(t)$ (occurring in the course of a vesicle trajectory) with transient changes in $n(t)$. This would in theory allow to determine the lifetime of a given linking state and hence the rates of transition between certain linking states.

The extraction of transition rates from SPT data required several analysis steps, which are described in detail below. First, the determined relationship between time-resolved vesicle diffusion coefficient $D$ and numbers of linking DNA-tethers $n$ was used to convert fluctuations in $D(t)$ into time-resolved changes in $n(t)$ (Figure 5 and Figure S5). This was done by a channelling approach, by assigning to all data points a linking number $n$ if the diffusion coefficients are within the interval defined by

$D_{\text {low }}=\frac{D_{*}}{n+0.5}$ and $D_{\text {up }}=\frac{D_{*}}{n-0.5}$.

Obviously, this conversion must be done using all trajectories, i.e., in contrast to the filtering procedure used for the derivation of the $D$ histograms, no trajectory was rejected during this data analysis step.

This conversion makes it possible to calculate the dependence of the probability $p_{\text {sur }}(n, \Delta t)$ for a trajectory to remain in its initial linking state $n$ on the observation time $\Delta t$. This was done by counting the number of trajectories that kept its initial linking state $n$ throughout the observation time $\Delta t$, referred to as $P_{\text {un }}(n, \Delta t)$, and the numbers $P_{+}(n, \Delta t)$ and $P_{-}(n, \Delta t)$ of trajectories that either increased or decreased $n$ within the observation time $\Delta t$. The "survival" probability $p_{\text {sur }}(n, \Delta t)$ was then calculated as

$$
p_{\text {sur }}(n, \Delta t)=\frac{P_{\text {un }}(n, \Delta t)}{P_{\text {un }}(n, \Delta t)+P_{+}(n, \Delta t)+P_{-}(n, \Delta t)} .
$$

For random transitions between the different linking states, a survival probability is expected to decay exponentially,

$$
p_{\text {sur }}(n, \Delta t) \propto \exp \left[-\left(k_{+}(n)+k_{-}(n)\right) \cdot \Delta t\right]
$$

where $k_{+}(n)$ and $k_{-}(n)$ are the rate constants of the transitions $n \rightarrow n+1$ and $n \rightarrow n-1$. 

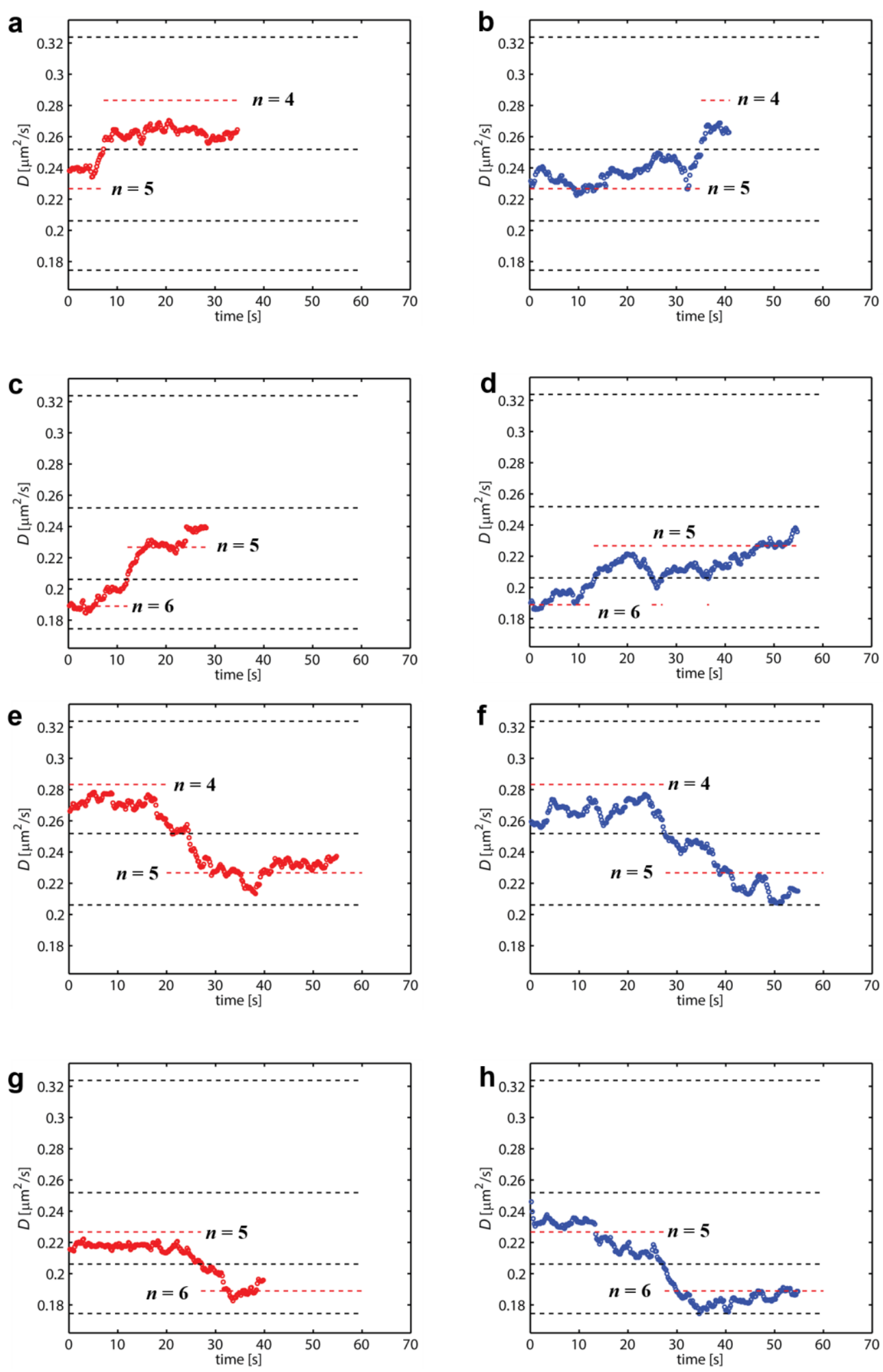

Figure S5: Conversion of the time-resolved diffusion coefficient (symbols) into the underlying linking state. Red dashed lines mark peak position observed in the diffusion coefficient histograms (Figure 3), corresponding to linking states as indicated in the plots, while black dashed lines correspond to the boundaries between these linking states. Trajectories cross sometimes the upper (a-d) or lower boundary $(\mathbf{e}-\mathbf{h})$, which is interpreted as a transition towards a linking state has lost (a-d) or gained (eh) one DNA-tether linking the tracked vesicle. Shown are the same trajectories as in Figure $5 \mathrm{~b}$ and c, but in independent plots allowing to see the assigned, time-resolved linking state (red dashed lines) in dependence of the observed time-resolved diffusion coefficient (symbols). 
Furthermore, the ratio of the numbers $P_{+}(n, \Delta t)$ and $P_{-}(n, \Delta t)$ of trajectories that either increased or decreased the number of DNA-linkers is equal to the ratio of these rate constants

$$
\frac{P_{+}(n, \Delta t)}{P_{-}(n, \Delta t)}=\frac{k_{+}(n)}{k_{-}(n)} .
$$

However, we observed that the survival probability extracted from SPT-based experiments usually deviated from the expected mono-exponential decay (Figure S6, symbols), which we attribute to distortions caused by the measurement process: (i) the fact that diffusion coefficient extraction requires collection of a certain number $N$ of data points from the trajectory (done in this work using a rolling window) and (ii) the intrinsic noise of random walks (whose variance decreases with increasing $N$; Equation S2).

The rolling window procedure, which corresponds to the application of a moving average filter, introduces a low pass-like behaviour upon relating the measured diffusion coefficient with the current linking state, as it averages out fluctuations that are faster than the cut-off frequency

$$
f_{\text {cut-off }}=\frac{0.4429}{\sqrt{N^{2}-1}} \cdot \frac{1}{\Delta T_{0}} .
$$

Hence, transitions rates that are faster than this cut-off frequency cannot be resolved by the extraction procedure and Equation S9 therefore gives the upper limit for extractable transition rates (lower dashed line in Figure S6).

The lower limit is determined by the intrinsic noise of random walks, which causes fluctuations of the extracted, time-resolved diffusive coefficient around the true value. The variance of these fluctuations is given by Equation S2 and decreases with increasing rolling window size $N$. Even if a vesicle remains in its initial binding state, there is (due to these fluctuations) always a non-zero probability to erroneously detect a transition into another state. This false-detection probability obviously increases with increasing standard deviation of the fluctuations (Equation S2) and observation time $\Delta t$ and defines the lowest resolvable transition rate, as it is the only source of (apparent) transitions for systems lacking any real transitions at all (upper dashed line in Figure S6).

These 2 processes transform the survival probability given by Equation S8 into a non-exponential decay (Figure S6, symbols) and fitting of these data points therefore requires numerical modelling of the measurement process using stochastic simulations. In brief, a single SPT trajectory (having 1 million steps) was generated based on a random walk algorithm. The respective diffusion coefficient was allowed 
to alternate between 3 different values, mimicking linking in the state of interest, $n$, and the 2 adjacent states, $n+1$ or $n-1$. The algorithm ensured that transitions between the different linking states occurred randomly with transition rates of $k_{+}(n)$, $k_{-}(n), k_{+}(n-1)$, and $k_{-}(n+1)$. The 2 rates, $k_{+}(n-1)$ and $k_{-}(n+1)$, ensure that the simulation returns after a certain observation time to the state of interest, $n$. As these rates do not influence the lifetime of the state of interest, they can be arbitrarily chosen and hence, in the simulation they were set equal to the total transition rate of the state of interest, $k_{+}(n)+k(n)$.

This created a SPT trajectory, whose diffusion coefficient fluctuated between 3 different states on a time scale set by $k_{+}(n)$ and $k_{-}(n)$. This single, long trajectory is subsequently split into pieces having 500 frames each, leading to a large set of shorter trajectories. As the transitions are randomly distributed, this is equivalent to simultaneously record multiple trajectories in a SPT experiment. These synthetic SPT trajectories were then analysed like real SPT data: 1. extraction of the time-resolved diffusion coefficient $D$ using a rolling window size of $N$ data points, 2. application of Equation $\mathrm{S} 7$ to relate $D(t)$ with the time-resolved number of linking DNA-tethers, $n(t)$, and 3. calculating the survival probability using Equation $\mathrm{S} 8$ and the transition rate ratio using Equation S10.

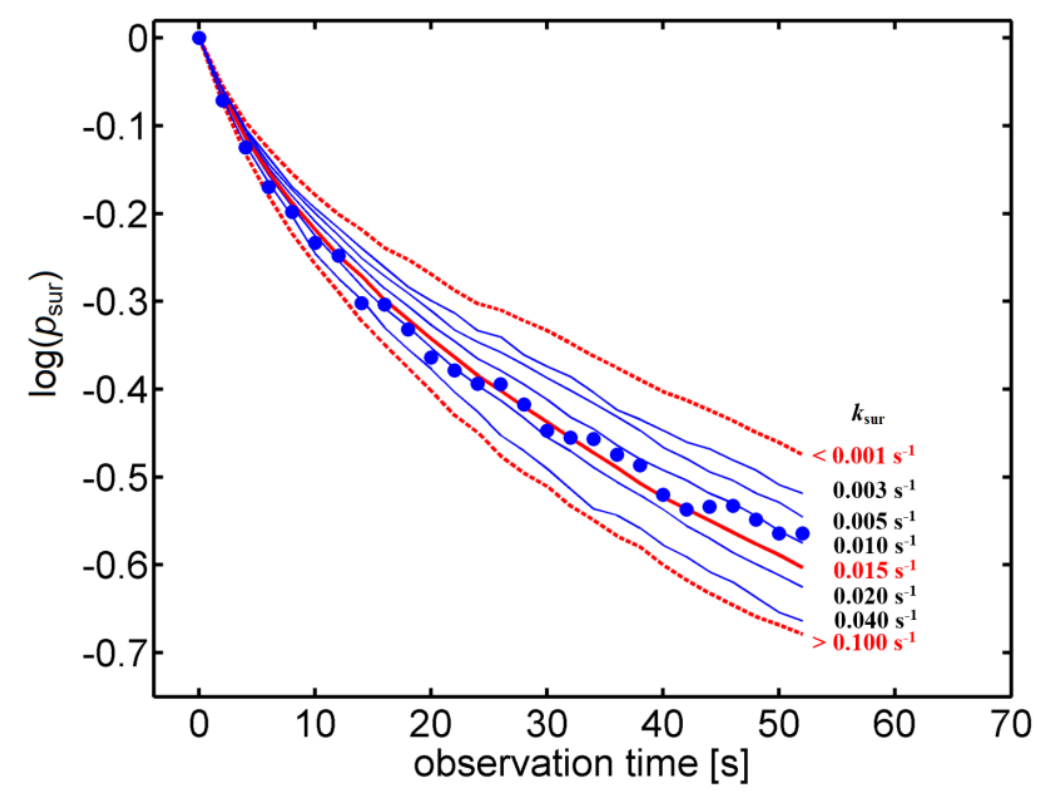

Figure S6: Probability $p_{\text {sur }}$ (Equation S8) of a vesicle to remain in its initial linking state in dependence of the observation time $\Delta t$ as extracted from SPT movies. The symbols give a representative example for vesicles that are initially linked by $n=5$ DNA-tethers (rolling window size $N=225$ frames), while the lines show survival probabilities $p_{\text {sur }}$ determined from numerical modelling of the measurement process as described in the text (the corresponding total transition rates $k_{\text {sur }}$ are indicated in the figure). The red line corresponds to the best fit of the numerics to the experimental data, yielding $k_{\text {sur }}=0.0147 \mathrm{~s}^{-1}$. The dashed blue lines indicate upper and lower limits for resolvable $k_{\text {sur }}$ that are caused by the low passlike behaviour of the rolling window (lower dashed line) and the intrinsic noise of random walks (upper dashed line). The extracted $k_{\text {sur }}$ is only reliable if the dashed lines are sufficiently separated so that the experimental data points do not touch them. 
The solid lines in Figure $\mathrm{S} 6$ give an example of survival probabilities $p_{\text {sur }}$ extracted from such stochastic simulations. As they depend only on the total transition rate constant, $k_{\text {sur }} \equiv k_{+}+k_{-}$, only this rate constant is indicated in the figure. Upper and lower limits for the resolvable total transition rate $k_{\text {sur }}$ are indicated by dashed lines in Figure S6. Variation of the simulated experimental parameters showed that these limits are formed by the above-mentioned processes, i.e., the lower limit of $k_{\text {sur }}$ is caused by the intrinsic noise of random walks and therefore depends mainly on the ratio of channel width to the SPT variance given by Equation $\mathrm{S} 2$, while the upper limit of $k_{\text {sur }}$ is created by the low pass-like behavior of the data extraction procedure, which averages out fluctuations that are faster than the cut-off frequency given by Equation S11. Hence, fitting of the summed transition rate $k_{\text {sur }}$ to data points is only meaningful if the data points fall within the area enclosed by the dashed lines in Figure S6.

As both limits depend on the size $N$ of the rolling window (Figure S7), the total transition rate $k_{\text {sur }}$ is always determined for different values of $N$. Fitted values of $k_{\text {sur }}$ were discarded from further data analysis, if the experimentally extracted survival probability entered the area beyond the lines defining the upper or lower limit of $k_{\text {sur }}$ (Figure S7d, left), indicating unreliably extraction of the summed transition rate. Approaching the lower limit of $k_{\text {sur }}$ (upper dashed lines in Figures S6 and S7) indicated insufficient accuracy in the determination of the diffusion coefficient, since apparent changes in the linking state were mainly caused by the intrinsic noise of random walkers (i.e., too high variance, Equation S2), suggesting that the accuracy could be improved by increasing $N$. On the other hand, the upper limit of $k_{\text {sur }}$ (lower dashed lines) was in these simulations touched if the transitions are faster than the cut-off frequency defined by Equation S11 (Figure S7d, left). Such fluctuations can only be accessed if the cut-off frequency is increased, which requires a decrease in $N$ or $\Delta N_{0}$ (Equation S11). These two constrains may lead to conflicting requirements on $N$, e.g. for fast transitions that require values of $N$ that are too small to obtain the accuracy required to resolve the involved change of the diffusion coefficient. As the spacing between adjacent peaks in the diffusion coefficient histogram decreases for increasing $n$, this situation is more likely to be observed for higher values of $n$ (Figure S7d, left).

This procedure allowed to extract the total transition rate $k_{\text {sur }}$ by fitting the results of stochastic simulations to the experimental data. Afterward, the relative distribution of $k_{\text {sur }}(n)$ among the rates $k_{+}(n)$ and $k_{-}(n)$ was determined by calculating the rate ratio $P_{+} / P_{-}$, Equation S10 (Figure S8). As discussed above, for random transitions one expects to observe a fixed rate ratio $P_{+} / P_{-}$that is independent of the observation interval $\Delta t$. In the stochastic simulations this behaviour is found only for small $\Delta t$ (Figure S8, lines), for which the probability to erroneously detect a transition (due to the intrinsic noise of random walks) is comparable for both transitions $n \rightarrow n+1$ and $n \rightarrow n-1$. However, the rate ratio increases monotonously for increasing $\Delta t$, suggesting that increasingly more transitions from $n \rightarrow n+1$ than from 
$n \rightarrow n-1$ are detected. This apparent increase in rate ratio is attributed to a distortion caused by the intrinsic noise of the random walk and the $1 / n$ - dependence of the peak position in the diffusion coefficient histogram, which decreases the width of the channels defined by Equation S7 for increasing $n$ (see left column of Figure S7). Hence, it is generally more likely to falsely detect a transition towards $n+1$ than to $n-1$, i.e., the rate ratio extracted by Equation $\mathrm{S} 10$ will increase with increasing $\Delta t$ (Figure S8). However, for small $\Delta t$ it is generally observed that the ratio $P_{+} / P$. extracted by Equation $S 10$ is constant and scales linearly with the actual rate ratio $k_{+} / k_{-}$between 0.2 and 2 (Figure S8, inset), which significantly simplifies the fitting procedure. This finally allowed decomposing the total transition rate $k_{\text {sur }}(n)$ into the individual rate constants $k_{+}(n)$ and $k_{-}(n)$ describing transition from $n$ to $n+1$ or $n-1$, respectively.

This procedure to extract transition rates from SPT data also offers a means to determine the requirements on the experimental parameters of a SPT experiment that needs to be fulfilled to determine transition rates that differ markedly from those associated with the system investigated in this work. Consider the case of a multivalent system, whose relation between (collective) diffusion coefficient $D(n)$ and binding state (i.e., number $n$ of binding linkers) follows the free draining model (Equation S6). A transition towards the binding state $n+1$ will be detected by the procedure, if the time-resolved diffusion coefficient of a trajectory drops below $D(n+0.5)$. In order to reliably resolve this transition, the accuracy in the determination of the diffusion coefficient must be sufficiently high, i.e., the standard deviation Equation $\mathrm{S} 2$ must be smaller than the difference $D(n)-D(n+0.5)$ :

$$
\begin{aligned}
& \frac{\sigma(n)}{D(n)}<\frac{D(n)-D(n+0.5)}{D(n)}=\frac{1}{2 n+1} \Rightarrow \sqrt{\frac{2}{3} \cdot \frac{N_{\mathrm{p}}}{N-N_{\mathrm{p}}}}<\frac{1}{2 n+1} \\
& \Rightarrow N>N_{\text {min }}(n)=\frac{2}{3} \cdot N_{\mathrm{p}} \cdot(2 n+1)^{2}
\end{aligned}
$$

Note that Equation $\mathrm{S} 12$ was derived under the assumptions of a $2 \mathrm{D}$ random walk and $N_{\mathrm{p}} \ll N$ (generally fulfilled in SPT experiments). ${ }^{2}$ This equation defines a lower limit $N_{\min }$ for the size of the rolling window that has to be exceeded to reliably resolve transitions from the binding state $n$. If the rolling window contains less frames than $N_{\min }$, the intrinsic fluctuations in the time-resolved diffusion coefficient will be larger than the difference $D(n)-D(n+0.5)$, thus strongly distorting the extraction of the transition rate. However, a rolling window of size $N$ is related to a low pass-like behaviour of the data analysis, averaging out all transitions that are faster than the cut-off frequency given by Equation $\mathrm{S} 11$. 

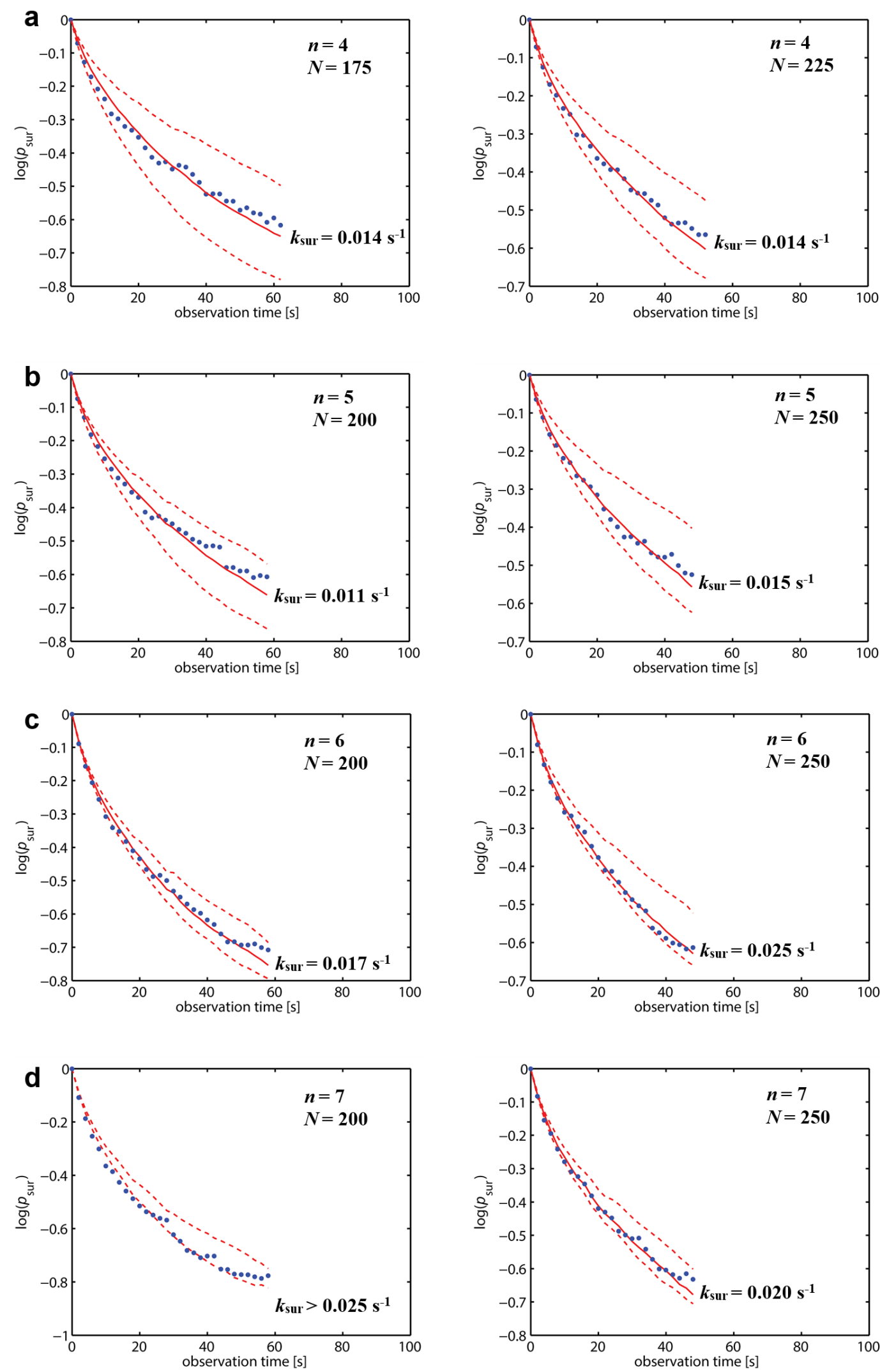

Figure S7: Representative examples for extracted probabilities $p_{\text {sur }}$ (Equation S8; $n$ ranging between 4 and 7 DNA-tethers) in dependence of the observation time $\Delta t$. Symbols show data from SPT movies, while solid lines give (similar to Figure S6) the best fits to the data points. The initial linking state $n$, the rolling window size $N$, and the fitted $k_{\text {sur }}$ are indicated in the figures. The dashed lines give upper and lower limits for resolvable $k_{\text {sur }}$ and depend on $n$ and $N$. Extracted values of $k_{\text {sur }}$ should be excluded from further analysis, if the data points touch the dashed lines (see e.g. Figure S7d, left). 


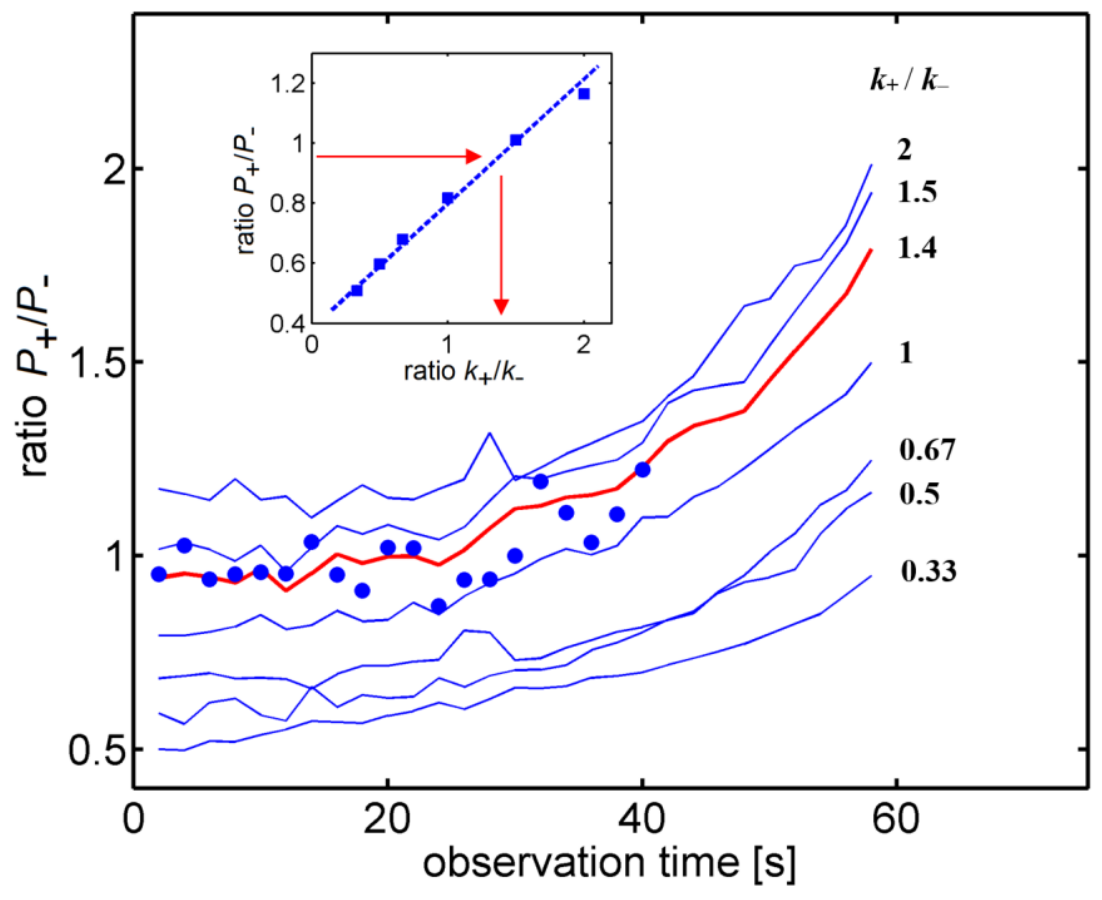

Figure S8: Dependence of the ratio $P_{+} / P_{-}$(Equation S10) on $\Delta t$, i.e., the number $P_{+}$of trajectories that increase their $n$ value during the observation time $\Delta t$ divided by the number $P_{-}$of trajectories that decrease $n$ during $\Delta t$, as extracted from SPT movies. The circles give a representative example for vesicles that are initially linked by $n=5$ DNA-tethers (rolling window size $N=200$ frames; $\left.k_{\text {sur }}=0.0114 \mathrm{~s}^{-1}\right)$, while the lines show survival probabilities $P_{+} / P_{-}$determined from numerical modelling of the measurement process using different rate ratios $k_{+} / k_{-}$(as indicated in the figure). It is established that for random transitions the ratio $P_{+} / P_{-}$equals the rate ratio $k_{+} / k_{-}$and is therefore independent of $\Delta t$. Experimentally (symbols) and numerically (lines) this is only observed for small $\Delta t$, as (due to the intrinsic noise of random walks) the false-detection rate for transitions that increase $n$ rises with $\Delta t$, leading to a systematic overestimation of $P_{+}$(see text for discussions). However, the plateau region of $P_{+} / P_{-}$for small $\Delta t$ generally scales linearly with $k_{+} / k_{-}$for rate ratios between 0.3 and 2 (inset), simplifying the fitting procedure used to decompose the total transition rate $k_{\text {sur }}$ into $k_{+}$ and $k$.

If $k_{\max }(n)$ denotes the fastest expected transition rate for the binding state $n$, the period $\Delta t_{0}$ between two frames have to be chosen such that the cut-off frequency given by Equation $\mathrm{S} 11$ exceeds $k_{\max }(n)$ :

$$
\begin{aligned}
& f_{\text {cut }- \text { off }}=\frac{0.4429}{\sqrt{N^{2}-1}} \cdot \frac{1}{\Delta t_{0}}>k_{\text {max }}(n) \\
& \Rightarrow \Delta t_{0}<\Delta t_{\max }(n, N)=\frac{0.4429}{\sqrt{N^{2}-1}} \cdot \frac{1}{k_{\max }(n)} \approx \frac{1}{2 \cdot N \cdot k_{\max }(n)} .
\end{aligned}
$$




\section{S8. Monte Carlo simulations of tether-mediated vesicle diffusion}

Our experimental results presented in the main part of the article indicate that diffusion of vesicles attached to a supported lipid bilayer via one or a few tethers (e.g., via fragments of DNA) can be described by using the so-called free draining model. This model implies that the vesicle diffusion is controlled by tethers which can migrate more or less independently inside the vesicle-bilayer contact region. The area of this region is considered to be much larger than the size of the tether crosssection. According to this model, the dependence of the diffusion coefficient on the number of tethers is determined by Equation S6 [or Equation (4) in the main text]. As already noted (in the main text), the conventional treatment of diffusion in the framework of the free draining model is somewhat heuristic, because instead of explicit description of diffusion the analysis is based on postulation of the relation between the friction coefficient of the whole aggregate with the friction coefficients of its parts, i.e., the analysis does not explicitly pay attention to the fact that the diffusion of tethers is spatially restricted. Here, we present direct Monte Carlo (MC) simulations of tether-mediated diffusion of vesicles.

In our analysis, we operate with the contact area, number of tethers, rate of diffusion of single tethers, and rate of diffusion of vesicles without shifts of tethers. The shape of attached vesicles during diffusion is considered to be close to that at equilibrium. The contact area is defined as the area where the distance between the vesicle membrane and the lipid bilayer is sufficiently small so that tethers can diffuse there without deformation of a vesicle. The radius of the contact area, $R$, depends primarily on the vesicle radius, number of tethers, their length and flexibility. Due to stochastic nature of this diffusion, tethers may sometimes be located asymmetrically with respect to the center of the contact area so that they are altogether somewhat shifted in one of the directions towards the boundary of the contact area. During such fluctuations, a vesicle may shift along the bilayer without shifts of tethers. Such shifts can be realized via rolling of a vesicle along the bilayer and are considered to be fast. Thus, the whole diffusion process is assumed to be limited by collective diffusion of tethers constrained at the contact area.

In our MC simulations, diffusion of each tether is described as a constrained random walk by introducing the jump rate, $v$, and radius, $\rho$. As already noticed, these jumps are constrained by the contact area. For vesicle jumps (without shifts of tethers), we use the jump rate, $w » v$, and the same jump radius, $\rho$ (in principle, one could choose different jump radiuses for jumps of a vesicle and tethers, but this detail does not influence the final conclusions). The latter jumps, associated with the shift of the center of the contact area, are constrained by the location of tethers.

With the specification above, our MC simulations are based on the standard Gillespie algorithm including the calculation of the total rate of all the possible steps, $w_{t}=\sum_{i} w_{i}$, and realization of one of the steps chosen with probability $w_{i} / w_{t}$. In our case, we have $w_{t}=n v+w$, and accordingly during a MC trial we choose either one of tethers with probability $n v /(n v+w)$ or a vesicle with probability $w /(n v+w)$. For a tether, 
the position after jump is selected at random around the initial position within a circle with radius $\rho$. If the selected position is within the contact area, a jump attempt is accepted. For a vesicle, the position after jump is chosen in the same way, and a trial is accepted if after displacement of the center of the contact area all the tethers remain there.

To describe the real time, the time should be changed after each $\mathrm{MC}$ trial by $|\ln (\varrho)| / w_{t}$, where $\varrho(0<\varrho \leq 1)$ is a random number. In general, however, the time increment can be chosen as $\Delta t=|\ln (\varrho)| \tau$, where $\tau$ is an arbitrary time interval. In our simulations, we use dimensionless time and choose $\tau=1 /(n+w / v)$ (with $w / v=10)$, so that on average the unit time corresponds to $n$ trials of the tether jumps. For $R$ and $\varrho$, we also use dimensionless units. Taking into account that for this model, the results are fairly insensitive to the ration $R / \rho$, we specify here these parameters as $R=10$ and $\rho=1$.

The vesicle diffusion coefficient is defined as $D=\left\langle r^{2}\right\rangle / 4 t$, where $\left\langle r^{2}\right\rangle$ is the mean square displacement of the center of the contact area during MC runs, and $t$ is the duration of a run. Our simulations indicate (Figure S9) that the dependence of the diffusion coefficient on the number of tethers is fairly accurately represented as $D \propto 1 / n$. The diffusion coefficient has been calculated using dimensionless units described above. In this units, the unit time corresponds to on one tether jump attempt on average. The unit length correspond to the maximum distance of tether jumps. The mean squared distance for one tether jump is $1 / 2$. Thus, the diffusion coefficient of single tethers without constrains is (1/2)/4=1/8=0.125. In Figure S9(a), one can see that the coefficient of vesicle diffusion with one tether is equal to 0.115 , i.e., only slightly smaller than that of single tethers. All these results are in agreement with Equation S6 [or Equation (4) in the main text]. 

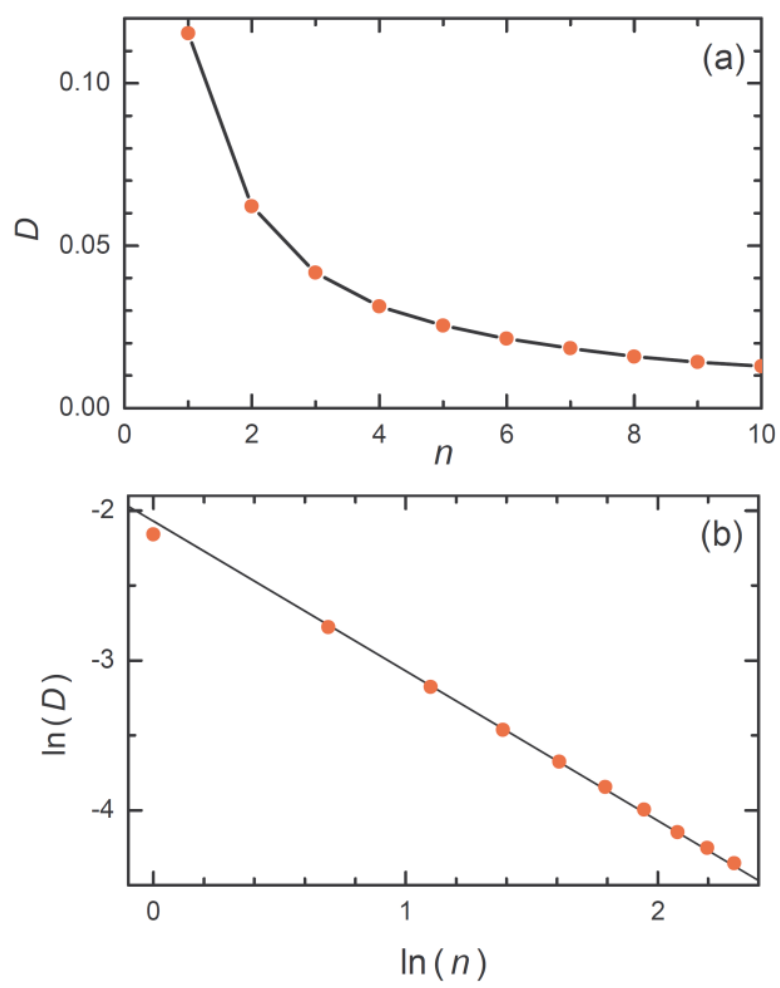

Figure S9: (a) Diffusion coefficient as a function of the number of tether. (b) The same data in the logarithmic coordinates. The straight line corresponds to $D \propto 1 / n$.

\section{S9. Width of $\boldsymbol{n}$ distribution}

The simplest estimate of the $n$ distribution is based on the assumption that the tethers are distributed at random and accordingly the distribution is Poissonian,

$$
P(n)=\frac{\langle n\rangle^{n} \exp (-\langle n\rangle)}{n !}
$$

where $\langle n\rangle$ is the average value. For $\langle n\rangle=3$, this distribution is rather wide (see Figure S10) and the scale of its FWHM is comparable with what we observe in Figure 2 of the main text. The observed FWHM (Figure 2) is, however, somewhat larger. Concerning this difference, one should take into account that the Poissonian distribution is expected to be applicable to vesicles in solution. For attached vesicles, the $n$ distribution is expected to be somewhat wider, because the vesicles with larger $n$ are more likely to be trapped. The scale of this effect can be estimated by assuming that the probability to be trapped is proportional to $n$. In this case, the simplest estimate of the $\mathrm{n}$ distribution is as follows

$\Gamma(n)=n P(n) /\langle n\rangle$

where $P(n)$ is the Poissonian distribution (S14). For $\langle n\rangle=3$, the FWHM of the latter distribution (Figure S10) is comparable to that we observe (Figure 2). 


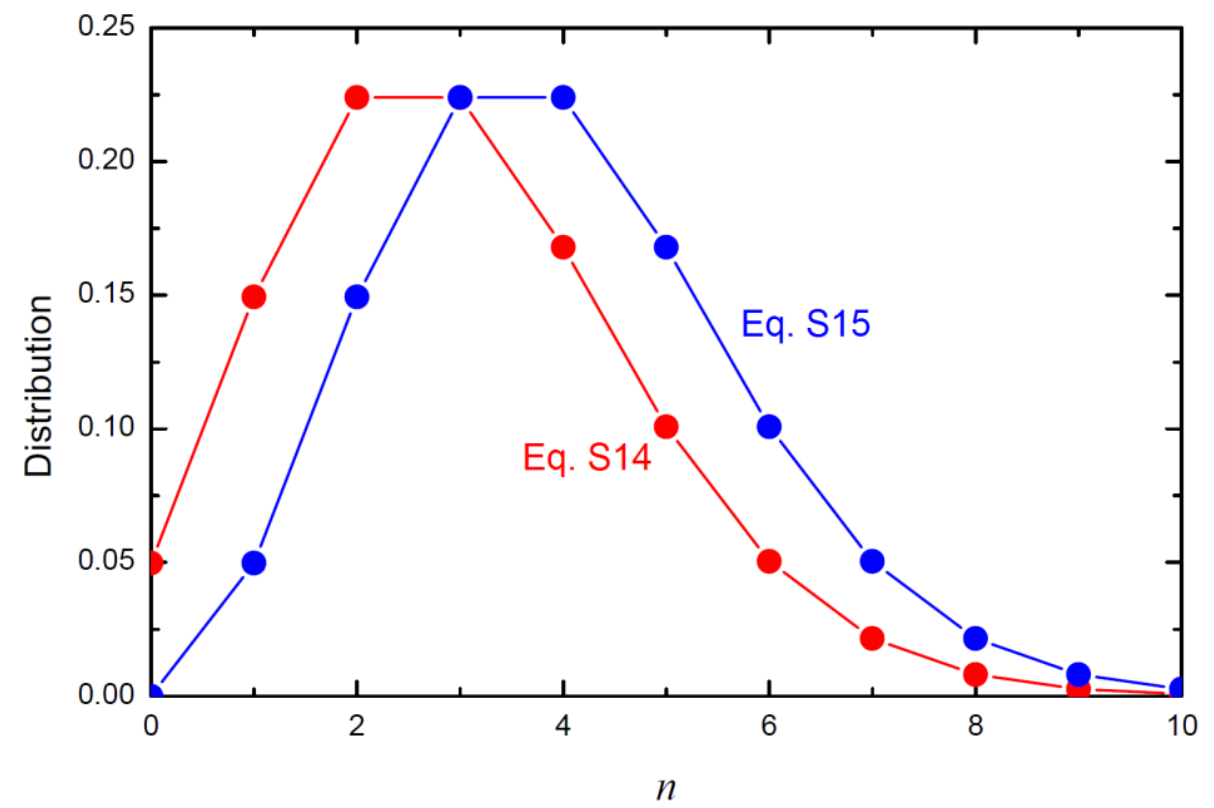

Figure S10: Distribution of the number of DNA-tethers in vesicles according to Equations S14 and S15 for $\langle n\rangle=3$.

\section{S10. Materials and methods}

Materials - POPC (1-palmitoyl-2-oleoyl-sn-glycero-3-phosphocholine), DSPEPEG(2k)biotin (1,2-distearoyl-sn-glycero-3-phosphoethanolamine-N[biotinyl(poly(ethylene glycol))-2000] and rhodamine-DOPE (1,2-dioleoylsn-glycero-3phosphoethanolamine- $\mathrm{N}$-(lissamine rhodamine $\mathrm{B}$ sulfonyl)) were obtained from Avanti Polar Lipids Inc. (Alabaster, AL). Cholesterol-terminated DNA-strands were obtained from Eurogentec S.A. (Seraing, Belgium) having the following sequences: 5'-TGG-ACA-TCA-GAA-ATA-AGG-CAC-GAC-GGA-CCC-chol-3' ( $(\alpha)$; 5'-chol-CCCTCC-GTC-GTG-CCT-3' $\quad\left(\alpha^{\prime}\right)$; $\quad$ 5'-TAT-TTC-TGA-TGT-CCA-AGC-CAC-GAG-TTCCCC-chol-3' ( $\left.\beta^{\prime}\right) ; \quad$ 5'-chol-CCC-GAA-CTC-GTG-GCT-3' ( $\beta$ ). Tris(hydroxymethyl)aminomethane hydrochloride (TRIS-HCl), sodium chloride and calcium chloride were obtained from Sigma Aldrich (Steinheim, Germany). If not otherwise stated, all solutions were prepared or diluted using a TRIS-HCl buffer consisting of $100 \mathrm{mM}$ Tris- $\mathrm{HCl}, 50 \mathrm{mM} \mathrm{NaCl}, 5 \mathrm{mM} \mathrm{CaCl}_{2}$ that was adjusted to $\mathrm{pH}=7.4$ using $\mathrm{HCl}$.

Vesicle Preparation - Lipid vesicles were prepared by the extrusion method as described earlier. ${ }^{14}$ Vesicles for supported lipid bilayer (SLB) formation consisted of $99.5 \mathrm{wt} \%$ POPC and $0.5 \mathrm{wt} \%$ DSPE-PEG(2k)biotin, while vesicles for single particle tracking were fluorescently labeled using the composition $98.5 \mathrm{wt} \%$ POPC, $0.5 \mathrm{wt} \%$ DSPE-PEG(2k)biotin and $1 \mathrm{wt} \%$ rhodamine-DOPE. Lipid films were dried in vacuum, hydrated by adding $1 \mathrm{~mL}$ of the Tris- $\mathrm{HCl}$ buffer, followed extruding the mixture 31 times through a polycarbonate membrane (pore size $100 \mathrm{~nm}$; Avanti Polar Lipids Inc., Alabaster, AL). 
PEGylated lipids were added to the vesicles, since recent studies indicated that these lipids enhance the fluidity of the SLB. ${ }^{15}$ The chosen concentration of PEGylated lipids ensures formation of a PEG cushion underneath the SLB, thereby reducing surface effects acting on the lipid diffusion. Moreover, addition of PEGylated lipids also decreased unspecific binding of vesicles to the SLB or sticking events, indicated by a large mobile fraction of linked vesicles observed in our experiments (> $90 \%)$.

TIRF-Microsopy - Total internal reflection fluorescence (TIRF) microscopy was conducted on an inverted Eclipse TE 2000 microscope (Nikon Corporation, Japan) that was equipped with a high-pressure mercury lamp, an Apo TIRF 60x oil objective (NA 1.49), and an Andor Neo CCD camera (Andor Technology, Belfast, Northern Ireland). A rhodamine filter set (TRITC, Semrock, Rochester, NY) was used, while focus drift was effectively reduced using the microscope's Perfect Focus System (PFS). Singe particle tracking (SPT) movies $(277 \mu \mathrm{m} \times 221 \mu \mathrm{m})$ contained 500 frames using a frame-to-frame period of $200 \mathrm{~ms}$ (= $5 \mathrm{fps})$.

SLB Formation and Vesicle Tethering - All TIRF experiments were conducted on glass microscope coverslips as surfaces, which were supplemented with a homemade PDMS well (volume $10 \mu \mathrm{L}) .{ }^{16}$ SLBs were formed by injecting POPC vesicles (lipid concentration $0.3 \mathrm{mg} / \mathrm{mL}$, average diameter $100 \mathrm{~nm}$; composition as indicated above), followed by rinsing (10 times) with Tris- $\mathrm{HCl}$ buffer. Vesicles were linked to the SLB using cholesterol-modified DNA strands as described earlier (Figure 1). ${ }^{17,} 18$ In brief, SLBs and vesicles were incubated separately with 2 different types of DNAstrands (Figure 1a), which carry a double-cholesterol group at one end that selfinserts the strands into the lipid bilayers (Figure 1b). Both types of DNA-strands share a conjugated single-stranded part at the other end (Figure 1a), which allows linking vesicles to the SLB via hybridization (Figure 1c).

In more detail, DNA-strands were incorporated into the SLB by incubation with SLB DNA-strand solution (concentration $300 \mathrm{nM}$ ) for $30 \mathrm{~min}$, followed by rinsing the well 10 times with $10 \mu \mathrm{L}$ of the TRIS-HCl buffer. In parallel, $12.5 \mu \mathrm{L}$ of labeled vesicles (lipid concentration $1 \mathrm{mg} / \mathrm{mL}$, average diameter $100 \mathrm{~nm}$; composition as indicated above) were mixed with either 8,16 , or $25 \mu \mathrm{L}$ of vesicle DNA-strand solution (concentration $50 \mathrm{nM}$ ), followed by further dilution with TRIS-HCl buffer to obtain a lipid concentration of $0.125 \mu \mathrm{g} / \mathrm{mL}$. Based on the average size of the vesicles (diameter $100 \mathrm{~nm}$ ) and a lipid head group area on the order of $0.5 \mathrm{~nm}^{2}$, these solutions contain approximately $7.8 \cdot 10^{5}$ vesicles/ $\mu \mathrm{L}$ that are mixed with either $24 \cdot 10^{5}, 48 \cdot 10^{5}$, or $72 \cdot 10^{5}$ vesicle DNA-strands/ $\mu \mathrm{L}$. Due to the high insertion efficiency of the employed DNA-tethers, ${ }^{19}$ which are (on the timescale of the experiment) irreversibly inserted into lipid bilayers, ${ }^{20}$ it is reasonable to assume that all vesicle DNA-strands become incorporated in these mixtures. Hence, these mixtures correspond to vesicles carrying on average approximately 3,6 , or 9 vesicle DNAstrands. After incubation, the SLB was washed again 10 times using the TRIS-HCl buffer and $10 \mu \mathrm{L}$ of the solution containing DNA-equipped SUVs (lipid concentration of $0.125 \mu \mathrm{g} / \mathrm{mL}$ corresponding to $1.3 \mathrm{nM}$ ) was added to the DNA-equipped SLB. 
Tethering of the SUVs (by hybridization of the complementary strands) was followed in real time using TIRF and blocked by rinsing the SLB 10 times with $10 \mu \mathrm{L}$ of the TRIS-HCl buffer, once a feasible surface coverage was obtained (on the order of 2 vesicles per $100 \mu \mathrm{m}^{2}$, reached during incubation times of few $10 \mathrm{sec}$ ). Afterward, single particle tracking (SPT) movies of the linked vesicles were recorded.

Application of the same approach to estimate the DNA-strand density incorporated into the SLB is connected to large errors, since in this case the effective surface area of the SLB is not known and since an unknown fraction of DNA-strands are taken up by the PDMS material. Control experiments involving different DNA-strand concentrations indicated, however, that incubation with 300 nM SLB DNA-strand solution leads to strand densities that are sufficient to hybridize all DNA-strands presented by the vesicles.

Data Analysis - All data analysis was done using home-made scripts written in MatLab (MathWorks, Natick, MA). Single particle tracking was implemented using local nearest-neighbor linking. ${ }^{21}$ Diffusion coefficients were calculated using the internal averaging procedure ${ }^{2}$ on a moving window of the data points and corrected for motion blur. ${ }^{22}$ The approach to exclude transition containing trajectories is described on page S2, while the extraction of transition rates is given on page S15. 


\begin{tabular}{l|l}
\hline$n$ & $D / \mu \mathrm{m}^{2} / \mathrm{s}$ \\
\hline 2 & $0.551 \pm 0.023$ \\
3 & $0.384 \pm 0.016$ \\
4 & $0.283 \pm 0.012$ \\
5 & $0.236 \pm 0.014$ \\
6 & $0.193 \pm 0.007$ \\
7 & $0.162 \pm 0.007$ \\
8 & $0.142 \pm 0.005$ \\
9 & $0.126 \pm 0.004$ \\
10 & $0.114 \pm 0.002$ \\
11 & $0.104 \pm 0.002$
\end{tabular}

Table S1: Refined peak diffusion coefficients versus number of linking DNA-tethers. 


\section{REFERENCES}

1. Codling, E. A.; Plank, M. J.; Benhamou, S. J R Soc Interface 2008, 5, (25), 813-834.

2. Saxton, M. J. Biophys J 1997, 72, (4), 1744-1753.

3. $\quad$ Michalet, X.; Berglund, A. J. Phys Rev E 2012, 85, (6).

4. $\quad$ Qian, H.; Sheetz, M. P.; Elson, E. L. Biophys J 1991, 60, (4), 910-21.

5. Saffman, P. G.; Delbruck, M. P Natl Acad Sci USA 1975, 72, (8), 3111-3113.

6. Flory, P. J., Principles of polymer chemistry. Cornell Univ. Press: Ithaca u.a., 1953; p XVI, $672 \mathrm{~S}$.

7. $\quad$ Kucik, D. F.; Elson, E. L.; Sheetz, M. P. Biophys J 1999, 76, (1), 314-322.

8. Jonsson, P.; Beech, J. P.; Tegenfeldt, J. O.; Höök, F. Langmuir 2009, 25, (11), 62796286.

9. Stanich, C. A.; Honerkamp-Smith, A. R.; Putzel, G. G.; Warth, C. S.; Lamprecht, A. K.; Mandal, P.; Mann, E.; Hua, T. A. D.; Keller, S. L. Biophys J 2013, 105, (2), 444454.

10. Honerkamp-Smith, A. R.; Machta, B. B.; Keller, S. L. Phys Rev Lett 2012, 108, (26).

11. Hormel, T. T.; Kurihara, S. Q.; Brennan, M. K.; Wozniak, M. C.; Parthasarathy, R. Phys Rev Lett 2014, 112, (18).

12. Weast, R. C.; Chemical Rubber Company (Cleveland Ohio), CRC handbook of chemistry and physics. 1. student ed.; CRC Press: Boca Raton, Fla., 1988.

13. Bunge, A.; Loew, M.; Pescador, P.; Arbuzova, A.; Brodersen, N.; Kang, J.; Dahne, L.; Liebscher, J.; Herrmann, A.; Stengel, G.; Huster, D. The Journal of Physical Chemistry B 2009, 113, (51), 16425-16434.

14. Macdonald, R. C.; Macdonald, R. I.; Menco, B. P. M.; Takeshita, K.; Subbarao, N. K.; Hu, L. R. Biochim Biophys Acta 1991, 1061, (2), 297-303.

15. Pace, H.; Nystrom, L. S.; Gunnarsson, A.; Eck, E.; Monson, C.; Geschwindner, S.; Snijder, A.; Höök, F. Analytical Chemistry 2015, 87, (18), 9194-9203.

16. Wahlsten, O.; Gunnarsson, A.; Nystrom, L. S.; Pace, H.; Geschwindner, S.; Höök, F. Langmuir 2015, 31, (39), 10774-10780.

17. Simonsson, L.; Jonsson, P.; Stengel, G.; Höök, F. Chemphyschem 2010, 11, (5), 1011-7.

18. Benkoski, J. J.; Höök, F. The Journal of Physical Chemistry B 2005, 109, (19), 9773-9.

19. Pfeiffer, I.; Höök, F. Analytical Chemistry 2006, 78, (21), 7493-7498.

20. Pfeiffer, I.; Höök, F. J Am Chem Soc 2004, 126, (33), 10224-10225.

21. Meijering, E.; Dzyubachyk, O.; Smal, I. Methods in Enzymology 2012, 504, 183-200.

22. Berglund, A. J. Phys Rev E 2010, 82, (1). 